\title{
The Automorphism Groups and Derivation Algebras of Two-Dimensional Algebras
}

\author{
Ahmed H ${ }^{1,2}$, Bekbaev $\mathrm{U}^{3}$ and Rakhimov $\mathrm{I}^{4 *}$ \\ ${ }^{1}$ Department of Mathematics, Faculty of Science, UPM, Selangor, Malaysia \\ ${ }^{2}$ Department of Mathematics, Faculty of Science, Taiz University, Taiz, Yemen \\ ${ }^{3}$ Department of Science in Engineering, Faculty of Engineering, IIUM, Kuala Lumpur, Malaysia \\ ${ }^{4}$ Institute for Mathematical Research (INSPEM), UPM, Serdang, Selangor, Malaysia
}

\section{Abstract}

The automorphism groups and derivation algebras of all two-dimensional algebras over algebraically closed fields are described.

Keywords: Algebra; Isomorphism; Structure constants; Automorphism; Derivation

MSC(2010): Primary: 17A36; 17B40; Secondary: 14R20; 14L30

\section{Introduction}

There is a long history and an extended study of the automorphism groups of algebras. Determining the full automorphism group of an algebra is generally an immensely difficult problem. For instance, the automorphism group of the polynomial ring of three variables is not yet understood, and a result in this direction is given by Shestakov and Umirbaev [1]. Since 1990, many researchers have successfully computed the automorphism groups of interesting infinite-dimensional noncommutative algebras, including certain quantum groups, generalized quantum Weyl algebras, skew polynomial rings and many more, the results are given [2-7], which is only a partial list. Recently, by using a rigidity theorem for quantum tori, Yakimov has proved the Andruskiewitsch-Dumas conjecture and the Launois-Lenagan conjecture $[8,9]$. A uniform approach to both the AndruskiewitschDumas conjecture and the Launois-Lenagan conjecture is provided in a preprint by Goodearl and Yakimov [10]. Authors [11] use the discriminant to determine the automorphism groups of some noncommutative algebras. Note that most of the results above are obtained for algebras over the fields of real or complex numbers. The study of the automorphism groups and derivation algebras is of a big interest due to the importance of them in the study of structure of algebras. This is one of the motivations to describe the automorphism groups and derivation algebras of all 2-dimensional algebras over any algebraically closed field.

Authors [12] have presented a complete list of isomorphism classes of two-dimensional algebras over algebraically closed fields, providing a list of canonical representatives of their structure constant's matrices. In the present paper we describe the groups of automorphisms and derivation algebras of all those listed algebras.

In fact, the automorphism groups of all 2-dimensional algebras have been given earlier [13]. In contrast to that we provide an explicit realization of the automorphism groups for the all listed canonical algebras [12].

The first part of this paper (Sections 2 and 3) is devoted to the description of the groups of automorphisms and the second part (Section 4) deals with the derivation algebras. In each case we consider problems over algebraically closed fields of characteristic not 2,3, characteristic 2 and characteristic 3 separately according to the classification results given [12].

\section{Preliminaries}

Let $\mathbb{F}$ be any field, for matrices $A=\left(a_{i j}\right), B$ over $\mathbb{F}$, as usual $A \otimes B$ stand for the block-matrix with blocks $\left(a_{i j} B\right)$.

\section{Definition 2.1}

A vector space $\mathbb{A}$ over $\mathbb{F}$ with multiplication $\cdot: \mathbb{A} \otimes \mathbb{A} \rightarrow \mathbb{A}$ given by $(\mathbf{u}, \mathbf{v}) \mapsto \mathbf{u} \cdot \mathbf{v}$ such that:

$(\alpha \mathbf{u}+\beta \mathbf{v}) \cdot \mathbf{w}=\alpha(\mathbf{u} \cdot \mathbf{w})+\beta(\mathbf{v} \cdot \mathbf{w}), \mathbf{w} \cdot(\alpha \mathbf{u}+\beta \mathbf{v})=\alpha(\mathbf{w} \cdot \mathbf{u})+\beta(\mathbf{w} \cdot \mathbf{v})$. whenever $\mathbf{u}, \mathbf{v}, \mathbf{w} \in \mathbb{A}$ and $\alpha, \beta \in \mathbb{F}$, is said to be an algebra.

\section{Definition 2.2}

Two algebras $\mathbb{A}$ and $\mathbb{B}$ are called isomorphic if there is an invertible linear map $\mathrm{f}: \mathbb{A} \rightarrow \mathbb{B}$ such that:

$$
\mathbf{f}\left(\mathbf{u} \cdot \cdot_{\mathbb{A}} \mathbf{v}\right)=\mathbf{f}(\mathbf{u}) \cdot_{\mathbb{B}} \mathbf{f}(\mathbf{v})
$$

whenever $\mathbf{u}, \mathbf{v} \in \mathbb{A}$.

\section{Definition 2.3}

An invertible linear map $\mathbf{f}: \mathbb{A} \rightarrow \mathbb{A}$ is said to be an automorphism if:

$$
\mathbf{f}(\mathbf{u} \cdot \mathbf{v})=\mathbf{f}(\mathbf{u}) \cdot \mathbf{f}(\mathbf{v})
$$

whenever $\mathbf{u}, \mathbf{v} \in \mathbb{A}$.

\section{Definition 2.4}

A linear map $\mathbf{d}: \mathbb{A} \rightarrow \mathbb{A}$ is said to be a derivation if:

$$
\mathbf{d}(\mathbf{u} \cdot \mathbf{v})=\mathbf{d}(\mathbf{u}) \cdot \mathbf{v}+\mathbf{u} \cdot \mathbf{d}(\mathbf{v})
$$

whenever $\mathbf{u}, \mathbf{v} \in \mathbb{A}$.

Let $\mathbb{A}$ be $m$-dimensional algebra over $\mathbb{F}$ and $e=\left(e^{1}, e^{2}, \ldots\right.$, $\left.e^{m}\right)$ its basis. Then the bilinear map is represented by a matrix $A=\left(A_{i j}^{k}\right) \in M\left(m \times m^{2} ; \mathbb{F}\right)$ as follows:

*Corresponding author: Rakhimov I, Institute for Mathematical Research (INSPEM), UPM, Serdang, Selangor, Malaysia, Tel: 03-8946 6831; E-mail: risamiddin@gmail.com

Received April 18, 2018; Accepted April 20, 2018; Published April 26, 2018

Citation: Ahmed H, Bekbaev U, Rakhimov I (2018) The Automorphism Groups and Derivation Algebras of Two-Dimensional Algebras. J Generalized Lie Theory Appl 12: 290. doi: 10.4172/1736-4337.1000290

Copyright: ( $2018 \mathrm{Ahmed} \mathrm{H}$, et al. This is an open-access article distributed under the terms of the Creative Commons Attribution License, which permits unrestricted use, distribution, and reproduction in any medium, provided the original author and source are credited. 


$$
\mathbf{u} \cdot \mathbf{v}=e A(u \otimes v),
$$

for $\mathbf{u}=e u, \mathbf{v}=e v$, where $u=\left(u_{1}, u_{2}, \ldots, u_{m}\right)^{T}$, and $v=\left(v_{1}, v_{2}, \ldots, v_{m}\right)^{T}$ are column coordinate vectors of $\mathbf{u}$ and $\mathbf{v}$, respectively. The matrix $A \in M\left(m \times m^{2} ; \mathbb{F}\right)$ defined above is called the matrix of structural constants (MSC) of $\mathbb{A}$ with respect to the basis $e$. Further we assume that a basis $e$ is fixed and we do not make a difference between the algebra $\mathbb{A}$ and its MSC $A$.

An automorphism g: $\mathbb{A} \rightarrow \mathbb{A}$ as an invertible linear map is represented by an invertible matrix $g \in G L(m ; \mathbb{F}): \mathbf{g}(\mathbf{u})=\mathbf{g}(e u)=e g u$. Due to:

$$
\mathbf{g}(\mathbf{u} \cdot \mathbf{v})=\mathbf{g}(e A(u \otimes v)=e g(A(u \otimes v))=e(g A)(u \otimes v),
$$

and

$$
\mathbf{g}(\mathbf{u}) \cdot \mathbf{g}(\mathbf{v})=(e g u) \cdot(e g v)=e A(g u \otimes g v)=e A g^{\otimes 2}(u \otimes v)
$$

the property $\mathbf{g}(\mathbf{u} \cdot \mathbf{v})=\mathbf{g}(\mathbf{u}) \cdot \mathbf{g}(\mathbf{v})$ is equivalent to:

$$
g A=A g^{\otimes 2} \text {. (1) }
$$

An derivation $\mathbf{d}: \mathbb{A} \rightarrow \mathbb{A}$ as a linear map is represented by a matrix $d \in M(m ; \mathbb{F})$ as follows $\mathbf{d}(\mathbf{u})=\mathbf{d}(e u)=e d u$. Due to:

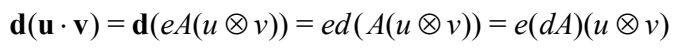

and

$\mathbf{d}(\mathbf{u}) \cdot \mathbf{v}+\mathbf{u} \cdot \mathbf{d}(\mathbf{v})=(e d u) \cdot(e v)+(e u) \cdot(e d v)=e A(d u \otimes v)+e A(u \otimes d v)=$ $e(A(d \otimes I)(u \otimes v)+A(I \otimes d)(u \otimes v))=e A((d \otimes I)+(I \otimes d))(u \otimes v)$

the property $\mathbf{d}(\mathbf{u} \cdot \mathbf{v})=\mathbf{d}(\mathbf{u}) \cdot \mathbf{v}+\mathbf{u} \cdot \mathbf{d}(\mathbf{v})$ is equivalent to:

$$
d A=A(d \otimes I+I \otimes d),
$$

where $I$ stands for the identity matrix.

If $e^{\prime}=\left(e^{\prime 1}, e^{\prime 2}, \ldots, e^{\prime m}\right)$ is another basis of $\mathbb{A}, e^{\prime} g=e$ with $g \in G=G L(m ; \mathbb{F})$, and $A^{\prime}$ is MSC of $\mathbb{A}$ with respect to $e^{\prime}$ then it is known that:

$$
A^{\prime}=g A\left(g^{-1}\right)^{\otimes 2}
$$

is valid. Thus, the isomorphism of algebras $\mathbb{A}$ and $\mathbb{B}$ over $\mathbb{F}$ given above now can be rewritten as follows.

\section{Definition 2.5}

Two $m$-dimensional algebras $\mathbb{A}, \mathbb{B}$ over $\mathbb{F}$, given by their matrices of structure constants $A, B$, are said to be isomorphic if $B=g A\left(g^{1}\right)^{2}$ holds true for some $g \in=G L(m ; \mathbb{F})$.

Further we consider only the case $m=2$ and for the simplicity we use:

$$
A=\left(\begin{array}{llll}
\alpha_{1} & \alpha_{2} & \alpha_{3} & \alpha_{4} \\
\beta_{1} & \beta_{2} & \beta_{3} & \beta_{4}
\end{array}\right)
$$

for MSC, where $\alpha_{1}, \alpha_{2}, \alpha_{3}, \alpha_{4}, \beta_{1}, \beta_{2}, \beta_{3}, \beta_{4}$ stand for any elements of $\mathbb{F}$.

Due to [1] we have the following classification theorems according to $\operatorname{Char}(\mathbb{F}) \neq 2,3, \operatorname{Char}(\mathbb{F})=2$ and $\operatorname{Char}(\mathbb{F})=3$ cases, respectively.

\section{Theorem 2.6}

Over an algebraically closed field $\mathbb{F}(\operatorname{Char}(\mathbb{F}) \neq 2$ and 3$)$, any nontrivial 2-dimensional algebra is isomorphic to only one of the following algebras listed by their matrices of structure constants:

$$
\begin{aligned}
& A_{1}(\mathbf{c})=\left(\begin{array}{cccc}
\alpha_{1} & \alpha_{2} & \alpha_{2}+1 & \alpha_{4} \\
\beta_{1} & -\alpha_{1} & -\alpha_{1}+1 & -\alpha_{2}
\end{array}\right) \text {, where } \mathbf{c}=\left(\alpha_{1}, \alpha_{2}, \alpha_{4}, \beta_{1}\right) \in \mathbb{F}^{4}, \\
& A_{2}(\mathbf{c})=\left(\begin{array}{cccc}
\alpha_{1} & 0 & 0 & 1 \\
\beta_{1} & \beta_{2} & 1-\alpha_{1} & 0
\end{array}\right) \simeq\left(\begin{array}{cccc}
\alpha_{1} & 0 & 0 & 1 \\
-\beta_{1} & \beta_{2} & 1-\alpha_{1} & 0
\end{array}\right) \text {, where } \mathbf{c}=\left(\alpha_{1}, \beta_{1}, \beta_{2}\right) \in \mathbb{F}^{3}, \\
& A_{3}(\mathbf{c})=\left(\begin{array}{cccc}
0 & 1 & 1 & 0 \\
\beta_{1} & \beta_{2} & 1 & -1
\end{array}\right) \text {, where } \mathbf{c}=\left(\beta_{1}, \beta_{2}\right) \in \mathbb{F}^{2}, \\
& A_{4}(\mathbf{c})=\left(\begin{array}{cccc}
\alpha_{1} & 0 & 0 & 0 \\
0 & \beta_{2} & 1-\alpha_{1} & 0
\end{array}\right) \text {, where } \mathbf{c}=\left(\alpha_{1}, \beta_{2}\right) \in \mathbb{F}^{2}, \\
& A_{5}(\mathbf{c})=\left(\begin{array}{cccc}
\alpha_{1} & 0 & 0 & 0 \\
1 & 2 \alpha_{1}-1 & 1-\alpha_{1} & 0
\end{array}\right), \text { where } \mathbf{c}=\alpha_{1} \in \mathbb{F}, \\
& A_{6}(\mathbf{c})=\left(\begin{array}{cccc}
\alpha_{1} & 0 & 0 & 1 \\
\beta_{1} & 1-\alpha_{1} & -\alpha_{1} & 0
\end{array}\right) \simeq\left(\begin{array}{cccc}
\alpha_{1} & 0 & 0 & 1 \\
-\beta_{1} & 1-\alpha_{1} & -\alpha_{1} & 0
\end{array}\right) \text {, where } \mathbf{c}=\left(\alpha_{1}, \beta_{1}\right) \in \mathbb{F}^{2}, \\
& A_{7}(\mathbf{c})=\left(\begin{array}{cccc}
0 & 1 & 1 & 0 \\
\beta_{1} & 1 & 0 & -1
\end{array}\right) \text {, where } \mathbf{c}=\beta_{1} \in \mathbb{F} \text {, } \\
& A_{8}(\mathbf{c})=\left(\begin{array}{cccc}
\alpha_{1} & 0 & 0 & 0 \\
0 & 1-\alpha_{1} & -\alpha_{1} & 0
\end{array}\right) \text {, where } \mathbf{c}=\alpha_{1} \in \mathbb{F}, \\
& A_{9}=\left(\begin{array}{cccc}
\frac{1}{3} & 0 & 0 & 0 \\
1 & \frac{2}{3} & -\frac{1}{3} & 0
\end{array}\right) \text {, } \\
& A_{10}=\left(\begin{array}{cccc}
0 & 1 & 1 & 0 \\
0 & 0 & 0 & -1
\end{array}\right) \text {, } \\
& A_{11}=\left(\begin{array}{cccc}
0 & 1 & 1 & 0 \\
1 & 0 & 0 & -1
\end{array}\right), \\
& A_{12}=\left(\begin{array}{llll}
0 & 0 & 0 & 0 \\
1 & 0 & 0 & 0
\end{array}\right)
\end{aligned}
$$

\section{Theorem 2.7}

Over an algebraically closed field $\mathbb{F}(\operatorname{Char}(\mathbb{F})=2)$, any non-trivial 2-dimensional algebra is isomorphic to only one of the following algebras listed by their matrices of structure constants:

$$
\begin{aligned}
& A_{1,2}(\mathbf{c})=\left(\begin{array}{cccc}
\alpha_{1} & \alpha_{2} & \alpha_{2}+1 & \alpha_{4} \\
\beta_{1} & -\alpha_{1} & -\alpha_{1}+1 & -\alpha_{2}
\end{array}\right), \text { where } \mathbf{c}=\left(\alpha_{1}, \alpha_{2}, \alpha_{4}, \beta_{1}\right) \in \mathbb{F}^{4}, \\
& A_{2,2}(\mathbf{c})=\left(\begin{array}{cccc}
\alpha_{1} & 0 & 0 & 1 \\
\beta_{1} & \beta_{2} & 1-\alpha_{1} & 0
\end{array}\right) \text {, where } \mathbf{c}=\left(\alpha_{1}, \beta_{1}, \beta_{2}\right) \in \mathbb{F}^{3}, \\
& A_{3,2}(\mathbf{c})=\left(\begin{array}{cccc}
0 & 1 & 1 & 0 \\
\beta_{1} & \beta_{2} & 1 & -1
\end{array}\right) \text {, where } \mathbf{c}=\left(\beta_{1}, \beta_{2}\right) \in \mathbb{F}^{2}, \\
& A_{4,2}(\mathbf{c})=\left(\begin{array}{cccc}
\alpha_{1} & 0 & 0 & 0 \\
0 & \beta_{2} & 1-\alpha_{1} & 0
\end{array}\right) \text {, where } \mathbf{c}=\left(\alpha_{1}, \beta_{2}\right) \in \mathbb{F}^{2}, \\
& A_{5,2}(\mathbf{c})=\left(\begin{array}{cccc}
\alpha_{1} & 0 & 0 & 0 \\
1 & 1 & 1-\alpha_{1} & 0
\end{array}\right) \text {, where } \mathbf{c}=\alpha_{1} \in \mathbb{F}, \\
& A_{6,2}(\mathbf{c})=\left(\begin{array}{cccc}
\alpha_{1} & 0 & 0 & 1 \\
\beta_{1} & 1-\alpha_{1} & -\alpha_{1} & 0
\end{array}\right) \text {, where } \mathbf{c}=\left(\alpha_{1}, \beta_{1}\right) \in \mathbb{F}^{2}, \\
& A_{7,2}(\mathbf{c})=\left(\begin{array}{cccc}
\alpha_{1} & 1 & 1 & 0 \\
0 & 1-\alpha_{1} & -\alpha_{1} & -1
\end{array}\right) \text {, where } \mathbf{c}=\alpha_{1} \in \mathbb{F}, \\
& A_{8,2}(\mathbf{c})=\left(\begin{array}{cccc}
\alpha_{1} & 0 & 0 & 0 \\
0 & 1-\alpha_{1} & -\alpha_{1} & 0
\end{array}\right) \text {, where } \mathbf{c}=\alpha_{1} \in \mathbb{F}, \\
& A_{9,2}=\left(\begin{array}{llll}
1 & 0 & 0 & 0 \\
1 & 0 & 1 & 0
\end{array}\right), \\
& A_{10,2}=\left(\begin{array}{cccc}
0 & 1 & 1 & 0 \\
0 & 0 & 0 & -1
\end{array}\right) \\
& A_{11,2}=\left(\begin{array}{cccc}
1 & 1 & 1 & 0 \\
0 & -1 & -1 & -1
\end{array}\right) \text {, } \\
& A_{12,2}=\left(\begin{array}{llll}
0 & 0 & 0 & 0 \\
1 & 0 & 0 & 0
\end{array}\right) .
\end{aligned}
$$

\section{Theorem 2.8}

Over an algebraically closed field $\mathbb{F}(\operatorname{Char}(\mathbb{F})=3)$, any non-trivial 
2-dimensional algebra is isomorphic to only one of the following algebras listed by their matrices of structure constant matrices:

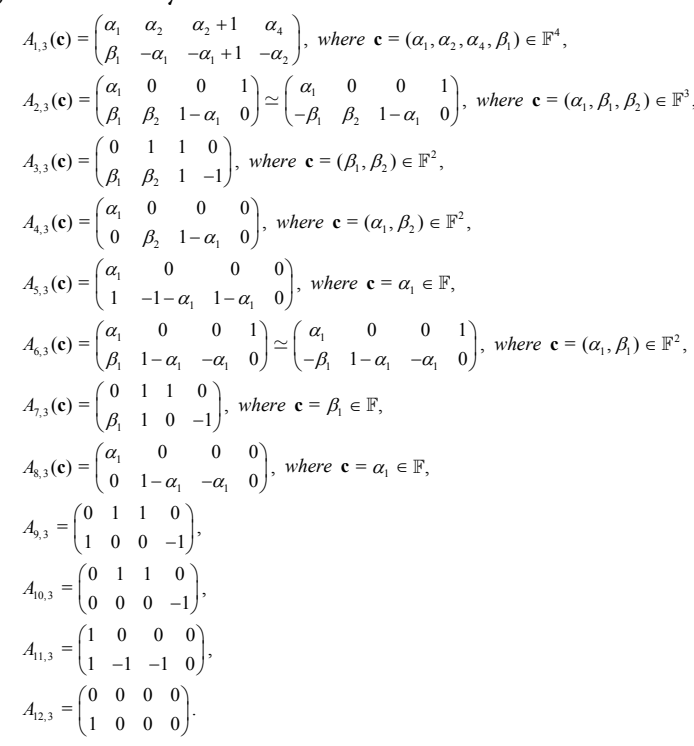

\section{The Groups of Automorphisms of 2-Dimensional Algebras}

Due to eqn. (1) for the group of automorphisms of an algebra $\mathbb{A}$ given by MSC $A \in M(2 \times 4 ; \mathbb{F})$ one has:

$$
\operatorname{Aut}(A)=\{g \in G L(2 ; \mathbb{F}): g A-A(g \otimes g)=0\} .
$$

Therefore further we look only for nonsingular solutions $g=\left(\begin{array}{ll}a & b \\ c & d\end{array}\right)$ of the equation $g A-A(g \otimes g)=0$. We consider this equation for each MSC $A$ from the theorems stated above separately and to avoid reparations we discuss only a few cases in details, the others can be understood easily from the discussions made. We use $\Delta$ for $\operatorname{det}(g)=a d-$ bc.

\section{Theorem 3.1}

The automorphism groups of the algebras listed in Theorem 2.6 are given as follows:

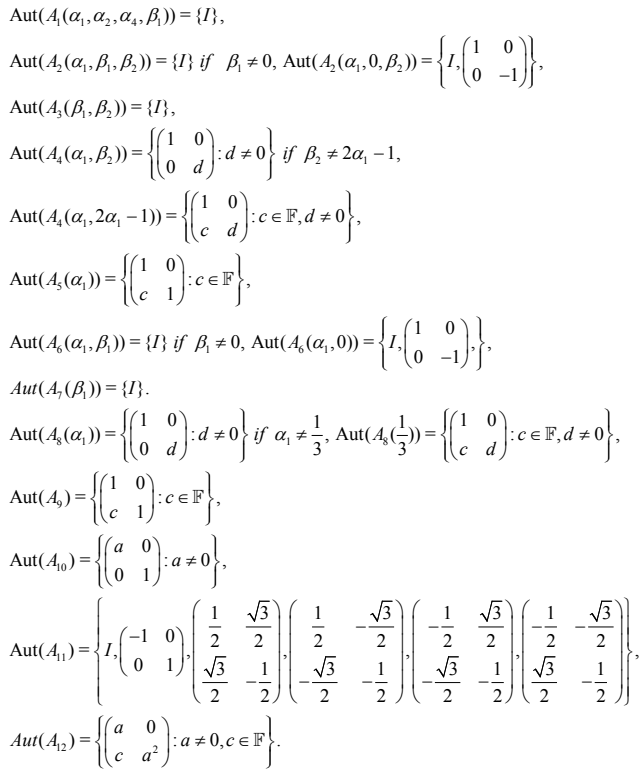

Proof. Let $A=A_{1}\left(\alpha_{1}, \alpha_{2}, \alpha_{4}, \beta_{1}\right)=\left(\begin{array}{cccc}\alpha_{1} & \alpha_{2} & \alpha_{2}+1 & \alpha_{4} \\ \beta_{1} & -\alpha_{1} & -\alpha_{1}+1 & -\alpha_{2}\end{array}\right)$

Due to eqn. (4) one has the system of equations:

$$
\begin{array}{ll}
-a c+a \alpha_{1}-a^{2} \alpha_{1}-2 a c \alpha_{2}-c^{2} \alpha_{4}+b \beta_{1} & =0, \\
-b c-b \alpha_{1}-a b \alpha_{1}+a \alpha_{2}-b c \alpha_{2}-a d \alpha_{2}-c d \alpha_{4} & =0, \\
a+b-a d-b \alpha_{1}-a b \alpha_{1}+a \alpha_{2}-b c \alpha_{2}-a d \alpha_{2}-c d \alpha_{4} & =0, \\
-b d-b^{2} \alpha_{1}-b \alpha_{2}-2 b d \alpha_{2}+a \alpha_{4}-d^{2} \alpha_{4} & =0, \\
-a c+c \alpha_{1}+2 a c \alpha_{1}+c^{2} \alpha_{2}-a^{2} \beta_{1}+d \beta_{1} & =0, \\
-b c+b c \alpha_{1}-d \alpha_{1}+a d \alpha_{1}+c \alpha_{2}+c d \alpha_{2}-a b \beta_{1} & =0, \\
c+d-a d+b c \alpha_{1}-d \alpha_{1}+a d \alpha_{1}+c \alpha_{2}+c d \alpha_{2}-a b \beta_{1} & =0, \\
-b d+2 b d \alpha_{1}-d \alpha_{2}+d^{2} \alpha_{2}+c \alpha_{4}-b^{2} \beta_{1} & =0,
\end{array}
$$

The eqns. (2) and (3), of the system (5) imply $a+b-a d+b c=0$, i.e., $b=\Delta-a$. Similarly the eqns. (6) and (7), of the system (5) imply $c+d-$ $a d+b c=0$, i.e., $d=\Delta-c$.

Therefore, $\Delta=a(\Delta-c)-c(\Delta-a)$, and this implies:

$\Delta(1-a+c)=0$.

Since $\Delta \neq 0$ we have $c=a-1$. The similar observation implies $d=b+1$. Therefore we get $g=\left(\begin{array}{cc}a & b \\ a-1 & b+1\end{array}\right)$, with $\Delta=a+b \neq 0$.

As a result the system (5) can be rewritten as follows:

$$
\begin{array}{cc}
a^{2}\left(1+\alpha_{1}+2 \alpha_{2}+\alpha_{4}\right)-a\left(1+\alpha_{1}+2 \alpha_{2}+2 \alpha_{4}\right)+\alpha_{4} & =0, \\
\alpha_{4}(a-1) & =0, \\
a^{2}\left(1-2 \alpha_{1}-\alpha_{2}+\beta_{1}\right)+a\left(-1+\alpha_{1}+2 \alpha_{2}\right)-\alpha_{2}+\alpha_{1}-\beta_{1} & =0, \\
\left(\alpha_{1}+2 \alpha_{2}\right)(a-1) & =0 .
\end{array}
$$

Note that if $(a, b)$ is a solution to the system and $b=0$ then $a=0$ or $a=1$. Indeed, the substitution 0 for $b$ in the system gives:

$$
\begin{array}{cc}
a^{2}\left(1+\alpha_{1}+2 \alpha_{2}+\alpha_{4}\right)-a\left(1+\alpha_{1}+2 \alpha_{2}+2 \alpha_{4}\right)+\alpha_{4} & =0, \\
\alpha_{4}(a-1) & =0, \\
a^{2}\left(1-2 \alpha_{1}-\alpha_{2}+\beta_{1}\right)+a\left(-1+\alpha_{1}+2 \alpha_{2}\right)-\alpha_{2}+\alpha_{1}-\beta_{1} & =0, \\
\left(\alpha_{1}+2 \alpha_{2}\right)(a-1) & =0 .
\end{array}
$$

Due to the first two equalities one has $a\left(1+\alpha_{1}+2 \alpha_{2}\right)(a-1)=0$, and therefore $a(a-1)=0$. In $a=0$ case $g$ is singular, and it is out of the consideration, but if $a=1$ then $g$ is the identity matrix. Therefore further it is assumed that $b(a+b) \neq 0$.

The eqns. (2) and (5) of the system of eqn. (6) imply;

$\left(3 \alpha_{1}+3 \alpha_{2}+\alpha_{4}-\beta_{1}\right) a b+\left(\alpha_{1}+2 \alpha_{2}+\alpha_{4}\right) a-\left(\alpha_{1}+2 \alpha_{2}+\alpha_{4}\right) b-\left(\alpha_{1}+2 \alpha_{2}+\alpha_{4}\right)=0$,

the eqns. (3) and (6) imply $b\left(b\left(3 \alpha_{1}+3 \alpha_{2}+\alpha_{4}-\beta_{1}\right)+2\left(\alpha_{1}+2 \alpha_{2}+\alpha_{4}\right)\right)=0$, i.e.,

$$
b\left(3 \alpha_{1}+3 \alpha_{2}+\alpha_{4}-\beta_{1}\right)+2\left(\alpha_{1}+2 \alpha_{2}+\alpha_{4}\right)=0 .
$$
i.e.,

The eqns. (2) and (3) imply $b(b+a)\left(1+\alpha_{1}+2 \alpha_{2}+\alpha_{4}\right)+b\left(\alpha_{1}+2 \alpha_{2}+\alpha_{4}\right)=0$,

$(b+a)\left(1+\alpha_{1}+2 \alpha_{2}+\alpha_{4}\right)+\left(\alpha_{1}+2 \alpha_{2}+\alpha_{4}\right)=b+a+(b+a+1)\left(\alpha_{1}+2 \alpha_{2}+\alpha_{4}\right)=0$.

But eqns. (7) and (8) imply:

$(b+a+1)\left(\alpha_{1}+2 \alpha_{2}+\alpha_{4}\right)=0$

and therefore due to eqn. (9) one has $a+b=0$, which is a contradiction. Therefore:

$\operatorname{Aut}\left(A_{1}\left(\alpha_{1}, \alpha_{2}, \alpha_{4}, \beta_{1}\right)\right)=\{I\}$. 

of $\mathbb{F}$.

Note that the proof of eqn. (10) does not depend on characteristics

Let $A=A_{2}\left(\alpha_{1}, \beta_{1}, \beta_{2}\right)=\left(\begin{array}{cccc}\alpha_{1} & 0 & 0 & 1 \\ \beta_{1} & \beta_{2} & 1-\alpha_{1} & 0\end{array}\right)$.

Due to eqn. (4) one has the system of equations:

$$
\begin{array}{cc}
-c^{2}+a \alpha_{1}-a^{2} \alpha_{1}+b \beta_{1} & =0, \\
-c d-a b \alpha_{1}+b \beta_{2} & =0, \\
b-c d-b \alpha_{1}-a b \alpha_{1} & =0, \\
a-d^{2}-b^{2} \alpha_{1} & =0, \\
-a c+c \alpha_{1}+a c \alpha_{1}-a^{2} \beta_{1}+d \beta_{1}-a c \beta_{2} & =0, \\
-b c+b c \alpha_{1}-a b \beta_{1}+d \beta_{2}-a d \beta_{2} & =0, \\
d-a d-d \alpha_{1}+a d \alpha_{1}-a b \beta_{1}-b c \beta_{2} & =0, \\
c-b d+b d \alpha_{1}-b^{2} \beta_{1}-b d \beta_{2} & =0 .
\end{array}
$$

From the eqns. (2) and (3) of the system (11) we get $b\left(1-\beta_{2}-\alpha_{1}\right)=0$. The following cases occur:

Case 1: $1-\alpha_{1}-\beta_{2} \neq 0$. Then $b=0$ and one gets $c=0$, so $a \alpha_{1}(1-a)=0$ and $d(1-a)\left(1-\alpha_{1}\right)=0$ due to the eqns. (1) and (7), respectively. Thus $a=1$. The eqn. (4) implies $d^{2}=1$ and $\beta_{1}\left(d-a^{2}\right)=0$ due to the eqn. (5). We get two cases:

Case 1.1. $\beta_{1}=0$. Then $d= \pm 1$, therefore $g$ is $\left(\begin{array}{cc}1 & 0 \\ 0 & \pm 1\end{array}\right)$

Case 1.2. $\beta_{1}$. Then $d=1$, so $g=I$.

Case 2: $\beta_{2}=1-\alpha_{1}$. If $b=0$ it is easy to see that $g$ equals $\left(\begin{array}{cc}1 & 0 \\ 0 & \pm 1\end{array}\right)$ if $\beta_{1}=0$, and $g=I$ if $\beta_{1} \neq 0$. Now one can assume that $b \neq 0$, so due to the eqns. (4) and (1) we have $\alpha_{1}=\frac{a-d^{2}}{b^{2}}, \quad \beta_{1}=\frac{c^{2} b^{2}-a^{2}+a d^{2}+a^{3}-a^{2} d^{2}}{b^{3}}$, respectively. The substitution them into eqn. (11) results the following system of equations:

$$
\begin{array}{cc}
-a-a^{2}+b^{2}-b c d+d^{2}+a d^{2} & =0, \\
a^{4}-a^{5}+a b c+2 a^{2} b c-2 a b^{3} c-a^{2} b^{2} c^{2}-a^{2} d+a^{3} d & \\
+b^{2} c^{2} d-a^{3} d^{2}+a^{4} d^{2}-b c d^{2}-2 a b c d^{2}+a d^{3}-a^{2} d^{3} & =0, \\
a^{3}-a^{4}+a b c-b^{3} c-a b^{2} c^{2}-a d+a^{2} d+b^{2} d-a b^{2} d & \\
-a^{2} d^{2}+a^{3} d^{2}-b c d^{2}+d^{3}-a d^{3} & =0, \\
a^{2}-a^{3}+b c-b^{2} c^{2}+2 a d-2 b^{2} d-a d^{2}+a^{2} d^{2}-2 d^{3} & =0 .
\end{array}
$$

Now we make use the eqns. (2) and (4) to get:

$a b c+a^{2} b c-2 a b^{3} c-a^{2} d-a^{3} d+2 a^{2} b^{2} d+b^{2} c^{2} d-b c d^{2}-2 a b c d^{2}+a d^{3}+a^{2} d^{3}=0$ that is:

$b c\left(a+a^{2}+b c d-d^{2}-a d^{2}\right)-a d\left(a+a^{2}+b c d-d^{2}-a d^{2}\right)-2 a b^{3} c+2 a^{2} b^{2} d=0$.

Then the eqn. (1) of the system gives:

$b^{3} c-a d b^{2}-2 a b^{3} c+2 a^{2} b^{2} d=0$,

$b^{3} c(1-2 a)-a d b^{2}(1-2 a)=b^{2}(1-2 a)(b c-a d)=0$

This implies $a=\frac{1}{2}$. The substitution it into the system (12) yields:

$$
-3+4 b^{2}-4 b c d+6 d^{2} \quad=0,
$$

$1+32 b c-32 b^{3} c-8 b^{2} c^{2}-4 d+32 b^{2} c^{2} d-2 d^{2}-64 b c d^{2}+8 d^{3}=0$,

$1+8 b c-16 b^{3} c-8 b^{2} c^{2}-4 d+8 b^{2} d-2 d^{2}-16 b c d^{2}+8 d^{3}=0$,

$$
1+8 b c-8 b^{2} c^{2}+8 d-16 b^{2} d-2 d^{2}-16 d^{3} \quad=0 .
$$

Due to the eqns. (1) and (2) of the system of eqn. (13) one gets:

$1+8 b c-8 b^{2} c^{2}-4 d-2 d^{2}-18 b c d^{2}+8 d^{3}=0$ and therefore taking into account the eqn. (3), one has $8 b^{2} c-4 b d=4 b(2 b c-d)=0$, i.e., $d=2 b c$. This implies $\Delta=a d-b c=b c-b c=0$, i.e., $g$ is singular. Therefore:

$$
\begin{aligned}
& \operatorname{Aut}\left(A_{2}\left(\alpha_{1}, \beta_{1}, \beta_{2}\right)\right)=\{I\} \text {, if } \beta_{1} \neq 0 \\
& \operatorname{Aut}\left(A_{2}\left(\alpha_{1}, 0, \beta_{2}\right)\right)=\left\{I,\left(\begin{array}{cc}
1 & 0 \\
0 & -1
\end{array}\right)\right\} \text {. } \\
& \text { Let } A=A_{3}\left(\beta_{1}, \beta_{2}\right)=\left(\begin{array}{cccc}
0 & 1 & 1 & 0 \\
\beta_{1} & \beta_{2} & 1 & -1
\end{array}\right) \text {. }
\end{aligned}
$$

Due to eqn. (4) one has the system of equations:

$$
\begin{array}{cl}
-2 a c+b \beta_{1} & =0, \\
a-b c-a d+b \beta_{2} & =0, \\
a+b-b c-a d & =0, \\
-b-2 b d & =0, \\
-a c+c^{2}-a^{2} \beta_{1}+d \beta_{1}-a c \beta_{2} & =0, \\
c-b c+c d-a b \beta_{1}+d \beta_{2}-a d \beta_{2} & =0, \\
c+d-a d+c d-a b \beta_{1}-b c \beta_{2} & =0, \\
-d-b d+d^{2}-b^{2} \beta_{1}-b d \beta_{2} & =0 .
\end{array}
$$

The eqns. (2) and (3) imply $b\left(1-\beta_{2}\right)=0$. The following cases may occur:

Case 1: $\beta_{2} 1$. In this case $b=0, c=0$ and $a(1-d)=0$, i.e., $d=1$. Therefore $a=1$ and $g=I$.

Case 2: $\beta_{2}=1$. If $b=0$ it is easy to see that $g=I$. If $b \neq 0$ then the eqn. (4) implies $d=-\frac{1}{2}$, hence eqn. (14) becomes:

$$
\begin{aligned}
-2 a c+b \beta_{1} & =0, \\
\frac{3 a}{2}+b-b c & =0, \\
-2 a c+c^{2}-\frac{\beta_{1}}{2}-a^{2} \beta_{1} & =0, \\
-\frac{1}{2}+\frac{a}{2}+\frac{c}{2}-b c-a b \beta_{1} & =0, \\
\frac{3}{4}+b-b^{2} \beta_{1} & =0 .
\end{aligned}
$$

The eqn. (2) of the system (15) implies $a=\frac{2}{3}(-b+b c)$ and since $b \neq 0$ one gets $\beta_{1}=\frac{2 a c}{b}$. Therefore, the system of eqn. (15) becomes:

$$
\begin{aligned}
\frac{2 c}{3}+\frac{4 b c}{3}+\frac{16 b^{2} c}{27}+\frac{c^{2}}{3}-\frac{4 b c^{2}}{3}-\frac{16 b^{2} c^{2}}{9}+\frac{16 b^{2} c^{3}}{9}-\frac{16 b^{2} c^{4}}{27} & =0, \\
-\frac{1}{2}-\frac{b}{3}+\frac{c}{2}-\frac{2 b c}{3}-\frac{8 b^{2} c}{9}+\frac{16 b^{2} c^{2}}{9}-\frac{8 b^{2} c^{3}}{9} & =0, \\
\frac{3}{4}+b+\frac{4 b^{2} c}{3}-\frac{4 b^{2} c^{2}}{3} & =0 .
\end{aligned}
$$

The identity:

$-\frac{1}{2}-\frac{b}{3}+\frac{c}{2}-\frac{2 b c}{3}-\frac{8 b^{2} c}{9}+\frac{16 b^{2} c^{2}}{9}-\frac{8 b^{2} c^{3}}{9}=\left(\frac{3}{4}+b+\frac{4 b^{2} c}{3}-\frac{4 b^{2} c^{2}}{3}\right)\left(\frac{2}{3} c-\frac{2}{3}\right)+\frac{b(1-4 c)}{3}$

shows that $g$ may be only singular as far as in this case $\Delta=\frac{b(1-4 c)}{3}$.

Let $A=A_{4}\left(\alpha_{1}, \beta_{2}\right)=\left(\begin{array}{cccc}\alpha_{1} & 0 & 0 & 0 \\ 0 & \beta_{2} & 1-\alpha_{1} & 0\end{array}\right)$.

Owing to eqn. (4) one has the system of equations: 


$$
\begin{array}{cc}
a \alpha_{1}-a^{2} \alpha_{1} & =0, \\
-a b \alpha_{1}+b \beta_{2} & =0, \\
b-b \alpha_{1}-a b \alpha_{1} & =0, \\
-b^{2} \alpha_{1} & =0, \\
-a c+c \alpha_{1}+a c \alpha_{1}-a c \beta_{2} & =0, \\
-b c+b c \alpha_{1}+d \beta_{2}-a d \beta_{2} & =0, \\
d-a d-d \alpha_{1}+a d \alpha_{1}-b c \beta_{2} & =0, \\
-b d+b d \alpha_{1}-b d \beta_{2} & =0 .
\end{array}
$$

It is easy to see that for this system $b=0$ (so $a \neq 0, d \neq 0$ due to $\Delta \neq 0$ ), $a \alpha_{1}(1-a)=0, d \beta_{2}(1-a)=0$, and $d(1-a)\left(1-\alpha_{1}\right)=0$ hence $a=1$. Therefore, $c\left(-1+2 \alpha_{1}-\beta_{2}\right)=0$ by the eqn. (5).

Case 1: $\beta_{2}=2 \alpha_{1}-1$. We get $g=\left(\begin{array}{ll}1 & 0 \\ c & d\end{array}\right)$, where $d \neq 0$. $d \neq 0$

Case 2: $\beta_{2} 2 \alpha_{1}-1$.In this case $c=0$ and we obtain $g=\left(\begin{array}{ll}1 & 0 \\ 0 & d\end{array}\right)$, where

Let $A=A_{5}\left(\alpha_{1}\right)=\left(\begin{array}{cccc}\alpha_{1} & 0 & 0 & 0 \\ 1 & 2 \alpha_{1}-1 & 1-\alpha_{1} & 0\end{array}\right)$.

From eqn. (4) we have the system of equations:

$$
\begin{array}{cl}
b+a \alpha_{1}-a^{2} \alpha_{1} & =0, \\
-b+2 b \alpha_{1}-a b \alpha_{1} & =0, \\
b-b \alpha_{1}-a b \alpha_{1} & =0, \\
-b^{2} \alpha_{1} & =0, \\
-a^{2}+d+c \alpha_{1}-a c \alpha_{1} & =0, \\
-a b-b c-d+a d+b c \alpha_{1}+2 d \alpha_{1}-2 a d \alpha_{1} & =0, \\
-a b+b c+d-a d-2 b c \alpha_{1}-d \alpha_{1}+a d \alpha_{1} & =0, \\
-b^{2}-b d \alpha_{1} & =0 .
\end{array}
$$

Here immediately we get $b=0, a=1, d=1$ and $g=\left(\begin{array}{ll}1 & 0 \\ c & 1\end{array}\right)$,

Let $A_{6}\left(\alpha_{1}, \beta_{1}\right)=\left(\begin{array}{cccc}\alpha_{1} & 0 & 0 & 1 \\ \beta_{1} & 1-\alpha_{1} & -\alpha_{1} & 0\end{array}\right)$.

Then eqn. (4) gives the system of equations:

$$
\begin{array}{cc}
-c^{2}+a \alpha_{1}-a^{2} \alpha_{1}+b \beta_{1} & =0, \\
b-c d-b \alpha_{1}-a b \alpha_{1} & =0, \\
-c d-b \alpha_{1}-a b \alpha_{1} & =0, \\
a-d^{2}-b^{2} \alpha_{1} & =0, \\
-a c+c \alpha_{1}+2 a c \alpha_{1}-a^{2} \beta_{1}+d \beta_{1} & =0, \\
d-a d+b c \alpha_{1}-d \alpha_{1}+a d \alpha_{1}-a b \beta_{1} & =0, \\
-b c+b c \alpha_{1}-d \alpha_{1}+a d \alpha_{1}-a b \beta_{1} & =0, \\
c-b d+2 b d \alpha_{1}-b^{2} \beta_{1} & =0 .
\end{array}
$$

It is easy to see from the system that $c=0, b=0$ and $a=1$. The eqns. (4) and (5), imply $d^{2}=1, \beta_{1}(d-1)=0$, therefore we have to consider the following two cases:

Case 1: $\beta_{1}=0$. We get $d= \pm 1$ and $g=\left(\begin{array}{cc}1 & 0 \\ 0 & \pm 1\end{array}\right)$.

Case 2: $\beta_{1} 0$. We obtain $d=1$ and $g=I$.

Let $A_{7}\left(\beta_{1}\right)=\left(\begin{array}{cccc}0 & 1 & 1 & 0 \\ \beta_{1} & 1 & 0 & -1\end{array}\right)$. Then:

$g A_{7}-A_{7}(g \otimes g)=\left(\begin{array}{cccc}-2 a c+b \beta_{1} & a+b-b c-a d & a-b c-a d & -b-2 b d \\ -a c+c^{2}-a^{2} \beta_{1}+d \beta_{1} & c+d-a d+c d-a b \beta_{1} & c-b c+c d-a b \beta_{1} & -d-b d+d^{2}-b^{2} \beta_{1}\end{array}\right)$

Thank to eqn. (4) we get $b=0, d=1, c=0$ and $a=1$, which implies $g=I$.
Let $A=A_{8}\left(\alpha_{1}\right)=\left(\begin{array}{cccc}\alpha_{1} & 0 & 0 & 0 \\ 0 & 1-\alpha_{1} & -\alpha_{1} & 0\end{array}\right)$. Then:

$g A_{8}-A_{8}(g \otimes g)=\left(\begin{array}{cccc}a \alpha_{1}-a^{2} \alpha_{1} & b-b \alpha_{1}-a b \alpha_{1} & -b \alpha_{1}-a b \alpha_{1} & -b^{2} \alpha_{1} \\ -a c+c \alpha_{1}+2 a c \alpha_{1} & d-a d+b c \alpha_{1}-d \alpha_{1}+a d \alpha_{1} & -b c+b c \alpha_{1}-d \alpha_{1}+a d \alpha_{1} & -b d+2 b d \alpha_{1}\end{array}\right)$

According to eqn. (4) one gets $b=0, d(1-a)=0$, that is $\mathrm{a}=1$, $c+3 c \alpha_{1}=0$. Again we consider two cases:

Case 1: $\alpha_{1}=\frac{1}{3}$. We get $g=\left(\begin{array}{ll}1 & 0 \\ c & d\end{array}\right)$, where $d \neq 0$.

Case 2: $\alpha_{1} \neq \frac{1}{3}$. Then $c=0$ and $g=\left(\begin{array}{ll}1 & 0 \\ 0 & d\end{array}\right)$, where $d \neq 0$. Therefore,

$\operatorname{Aut}\left(A_{8}\left(\frac{1}{3}\right)\right)=\left\{\left(\begin{array}{ll}1 & 0 \\ c & d\end{array}\right): d \neq 0\right\}$.

$\operatorname{Aut}\left(A_{8}\left(\frac{1}{3}\right)\right)=\left\{\left(\begin{array}{ll}1 & 0 \\ c & d\end{array}\right): d \neq 0\right\}$.

Let $A=A_{9}=\left(\begin{array}{cccc}\frac{1}{3} & 0 & 0 & 0 \\ 1 & \frac{2}{3} & -\frac{1}{3} & 0\end{array}\right)$. Then:

$g A_{9}-A_{9}(g \otimes g)=\left(\begin{array}{cccc}\frac{a}{3}-\frac{a^{2}}{3}+b & \frac{2 b}{3}-\frac{a b}{3} & -\frac{b}{3}-\frac{a b}{3} & -\frac{b^{2}}{3} \\ -a^{2}+\frac{c}{3}-\frac{a c}{3}+d & -a b+\frac{b c}{3}+\frac{2 d}{3}-\frac{2 a d}{3} & -a b-\frac{2 b c}{3}-\frac{d}{3}+\frac{a d}{3} & -b^{2}-\frac{b d}{3}\end{array}\right)$.

Owing to eqn. (4) one has $b=0, a=1, d=1$ and $g=\left(\begin{array}{ll}1 & 0 \\ c & 1\end{array}\right)$.

Let $A=A_{10}=\left(\begin{array}{cccc}0 & 1 & 1 & 0 \\ 0 & 0 & 0 & -1\end{array}\right)$. Then:

$g A_{10}-A_{10}(g \otimes g)=\left(\begin{array}{cccc}-2 a c & a-b c-a d & a-b c-a d & -b-2 b d \\ c^{2} & c+c d & c+c d & -d+d^{2}\end{array}\right)$.

Due to eqn. (4) it is easy to see that $c=0, d=1$ and $b=0$ i.e., $g=\left(\begin{array}{ll}a & 0 \\ 0 & d\end{array}\right)$. where $a \neq 0$

Let $A=A_{11}=\left(\begin{array}{cccc}0 & 1 & 1 & 0 \\ 1 & 0 & 0 & -1\end{array}\right)$. Then:

$g A_{11}-A_{11}(g \otimes g)=\left(\begin{array}{cccc}b-2 a c & a-b c-a d & a-b c-a d & -b-2 b d \\ -a^{2}+c^{2}+d & -a b+c+c d & -a b+c+c d & -b^{2}-d+d^{2}\end{array}\right)$.

The eqn. (4) gives $b(1+2 d)=0$. The following cases may occur:

Case 1: $b=0$. Then one has $c=0, d=1, a= \pm 1$ and $g=\left(\begin{array}{cc} \pm 1 & 0 \\ 0 & 1\end{array}\right)$.

Case 2: $d=-\frac{1}{2}$. In this case $b= \pm \frac{\sqrt{3}}{2}, a= \pm \frac{1}{2}$ and $c=\frac{b}{2 a}$. 
Case 2.1. $a=\frac{1}{2}$. Then $c= \pm \frac{\sqrt{3}}{2}$ and $g=\left(\begin{array}{cc}\frac{1}{2} & \pm \frac{\sqrt{3}}{2} \\ \pm \frac{\sqrt{3}}{2} & -\frac{1}{2}\end{array}\right)$.

Case 2.2. $a=-\frac{1}{2}$. Then $c=\mp \frac{\sqrt{3}}{2}$ and $g=\left(\begin{array}{cc}-\frac{1}{2} & \pm \frac{\sqrt{3}}{2} \\ \mp \frac{\sqrt{3}}{2} & -\frac{1}{2}\end{array}\right)$.

Let $A=A_{12}=\left(\begin{array}{cccc}0 & 0 & 0 & 0 \\ 1 & 0 & 0 & 0\end{array}\right)$. Then:

$$
g A_{12}-A_{12}(g \otimes g)=\left(\begin{array}{cccc}
b & 0 & 0 & 0 \\
-a^{2}+d & -a b & -a b & -b^{2}
\end{array}\right) .
$$

Due to eqn. (4) one has $g=\left(\begin{array}{cc}1 & 0 \\ c & a^{2}\end{array}\right)$, where $a \neq 0$.

Here are the corresponding results in the cases of characteristic 2 and 3. The proof is similar to that of the case of characteristic not 2 and 3 .

\section{Theorem 3.2}

The automorphism groups of the algebras listed in Theorem 2.7 are given as follows:

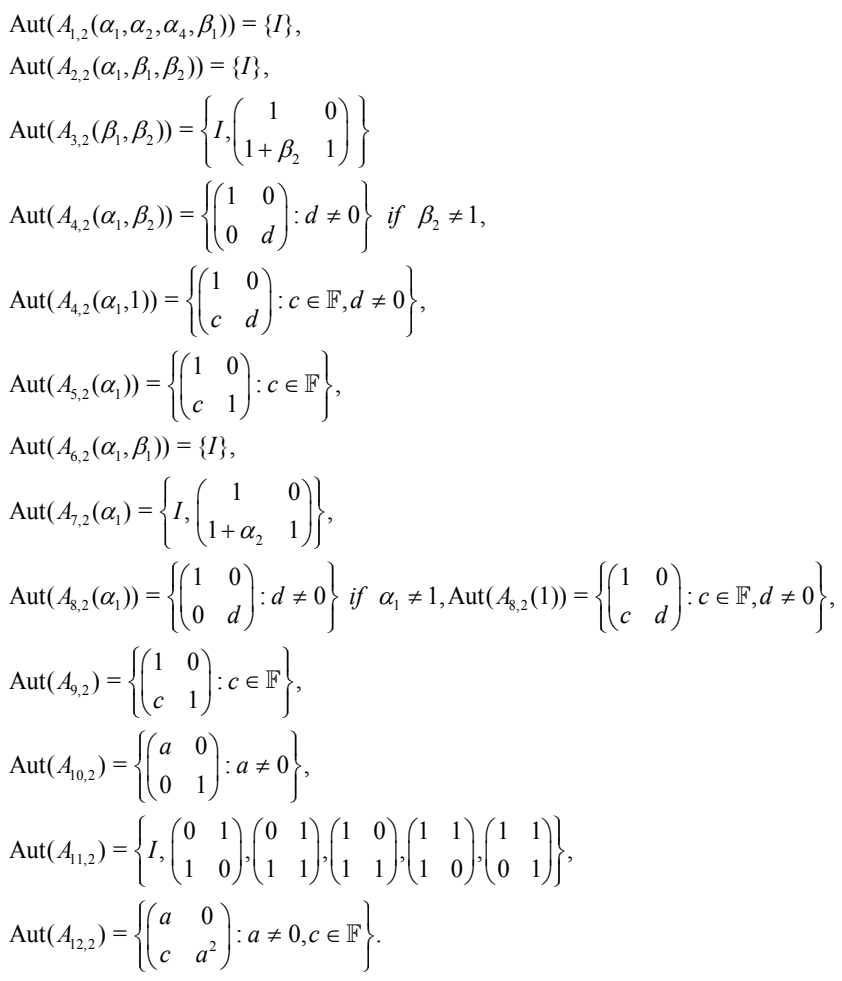

\section{Theorem 3.3}

The automorphism groups of the algebras listed in Theorem 2.8 are given as follows:

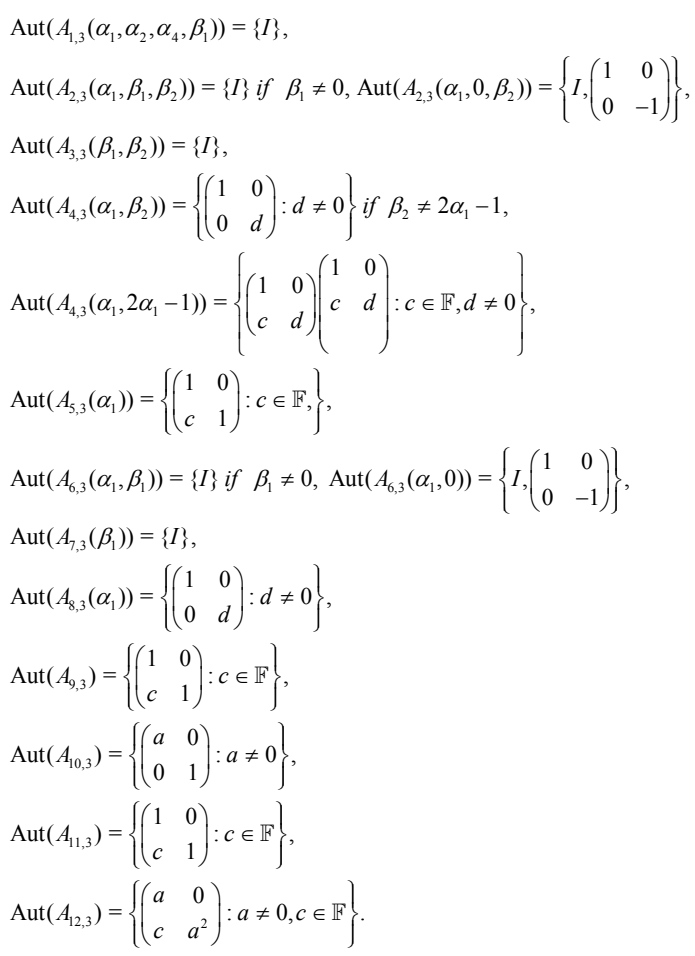

\section{Remark 3.4}

Another interpretation of Theorem 3.1 (Theorem 3.2, Theorem 3.3) is the description of stabilizers of the matrices listed in Theorem 2.6 (respectively, Theorem 2.7, Theorem 2.8), with respect to the action (3).

\section{Derivations of 2-dimensional Algebras}

If $\mathbb{A}$ is an algebra given by MSC $A$ then, due to eqn. (2) the algebra of its derivations $\operatorname{Der}(A)$ is represented as follows:

$\operatorname{Der}(A)=\{D \in M(2 ; \mathbb{F}): A(D \otimes I+I \otimes D)-D A=0\}$.

Further we use the notation $D=\left(\begin{array}{ll}a & b \\ c & d\end{array}\right)$.

One of the main results of this section is given in the following theorem.

\section{Theorem 4.1}

The derivations of all algebra structures on 2-dimensional vector space over an algebraically closed field $\mathbb{F}$ of characteristic not 2,3 are given as follows.

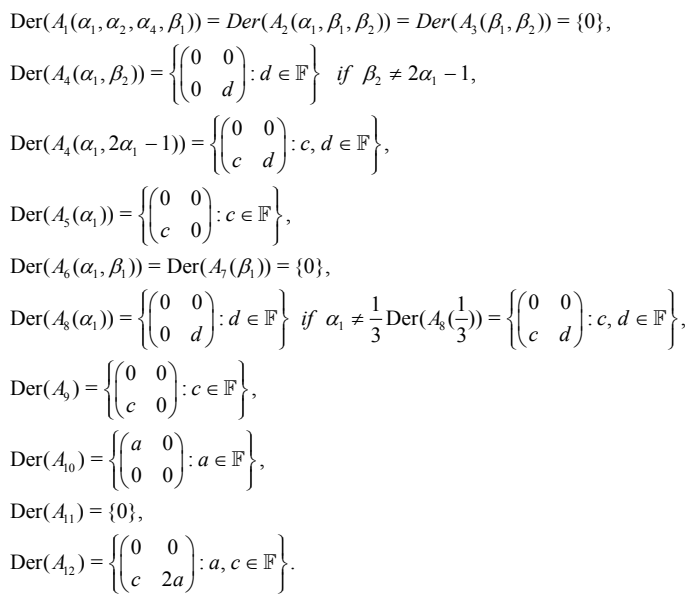


Proof. Let $A=A_{1}\left(\alpha_{1}, \alpha_{2}, \alpha_{4}, \beta_{1}\right)=\left(\begin{array}{cccc}\alpha_{1} & \alpha_{2} & \alpha_{2}+1 & \alpha_{4} \\ \beta_{1} & -\alpha_{1} & -\alpha_{1}+1 & -\alpha_{2}\end{array}\right)$.

Then the equality $A_{1}\left(\alpha_{1}, \alpha_{2}, \alpha_{4}, \beta_{1}\right)(D \otimes I+I \otimes D)-D A_{1}\left(\alpha_{1}, \alpha_{2}, \alpha_{4}, \beta_{1}\right)=0$ is equivalent to the following system of equations:

$$
\begin{array}{cl}
c+a \alpha_{1}+2 c \alpha_{2}-b \beta_{1} & =0, \\
c-3 c \alpha_{1}+2 a \beta_{1}-d \beta_{1} & =0, \\
2 b \alpha_{1}+d \alpha_{2}+c \alpha_{4} & =0, \\
-a \alpha_{1}-2 c \alpha_{2}+b \beta_{1} & =0, \\
-b+d+2 b \alpha_{1}+d \alpha_{2}+c \alpha_{4} & =0, \\
a-c-a \alpha_{1}-2 c \alpha_{2}+b \beta_{1} & =0, \\
b+3 b \alpha_{2}-a \alpha_{4}+2 d \alpha_{4} & =0, \\
b-2 b \alpha_{1}-d \alpha_{2}-c \alpha_{4} & =0 .
\end{array}
$$

The eqns. (3) and (8) of the system of equations above imply $b=0$, the eqns. (1) and (6) imply $a=0$, the eqns. (3) and (5) imply $d=0$ and the eqns. (1) and (4) imply $c=0$, therefore we get $D=0$.

Let $A=A_{2}\left(\alpha_{1}, \beta_{1}, \beta_{2}\right)=\left(\begin{array}{cccc}\alpha_{1} & 0 & 0 & 1 \\ \beta_{1} & \beta_{2} & 1-\alpha_{1} & 0\end{array}\right)$.

Then the eqn. (16) is equivalent to the system of equations:

$$
\begin{array}{cl}
a \alpha_{1}-b \beta_{1} & =0, \\
c-2 c \alpha_{1}+2 a \beta_{1}-d \beta_{1}+c \beta_{2} & =0, \\
c+b \alpha_{1}-b \beta_{2} & =0, \\
b \beta_{1}+a \beta_{2} & =0, \\
-b+c+2 b \alpha_{1} & =0, \\
a-a \alpha_{1}+b \beta_{1} & =0, \\
-a+2 d & =0, \\
b-c-b \alpha_{1}+b \beta_{2} & =0 .
\end{array}
$$

The eqns. (1) and (6) imply $a=0$, then the eqn. (7) gives $d=0$, the eqns. (3) and (8) imply $b=0$ and then due to the eqn. (3) one gets $c=0$, therefore one has $D=0$.

$$
\text { Let } A=A_{3}\left(\beta_{1}, \beta_{2}\right)=\left(\begin{array}{cccc}
0 & 1 & 1 & 0 \\
\beta_{1} & \beta_{2} & 1 & -1
\end{array}\right) \text {. }
$$

Then due to eqn. (16) one has the system of equations:

$$
\begin{array}{cl}
2 c-b \beta_{1} & =0, \\
c+2 a \beta_{1}-d \beta_{1}+c \beta_{2} & =0, \\
d-b \beta_{2} & =0, \\
-2 c+b \beta_{1}+a \beta_{2} & =0, \\
-b+d & =0, \\
a-2 c+b \beta_{1} & =0, \\
3 b & =0, \\
b-d+b \beta_{2} & =0 .
\end{array}
$$

The eqns. (5) and (7) imply $b=d=0$, the eqn. (1) gives $c=0$, the eqn. (6) implies $a=0$, hence $D=0$.

Let $A=A_{4}\left(\alpha_{1}, \beta_{2}\right)=\left(\begin{array}{cccc}\alpha_{1} & 0 & 0 & 0 \\ 0 & \beta_{2} & 1-\alpha_{1} & 0\end{array}\right)$.

Then the eqn. (16) is equivalent to the system of equations:

$$
\begin{array}{cl}
a \alpha_{1} & =0, \\
c-2 c \alpha_{1}+c \beta_{2} & =0, \\
b \alpha_{1}-b \beta_{2} & =0, \\
a \beta_{2} & =0, \\
-b+2 b \alpha_{1} & =0, \\
a-a \alpha_{1} & =0, \\
b-b \alpha_{1}+b \beta_{2} & =0 .
\end{array}
$$

The eqns. (3) and (7) imply $\mathrm{b}=0$, the eqns. (1) and (6) imply $a=0$. Therefore, $c\left(1-2 \alpha_{1}+\beta_{2}\right)=0$. If $\beta_{2} 2 \alpha_{1}-1$ one gets $D=\left(\begin{array}{ll}0 & 0 \\ 0 & d\end{array}\right)$, if $\beta_{2}=2 \alpha_{1}-1$ we obtain $D=\left(\begin{array}{ll}0 & 0 \\ c & d\end{array}\right)$.

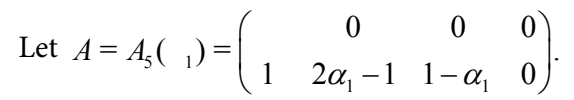

Then due to eqn. (16) one has the system of equations:

$$
\begin{array}{cc}
-b+a \alpha_{1} & =0, \\
2 a-d & =0, \\
b-b \alpha_{1} & =0, \\
-a+b+2 a \alpha_{1} & =0, \\
-b+2 b \alpha_{1} & =0, \\
a+b-a \alpha_{1} & =0, \\
b \alpha_{1} & =0 .
\end{array}
$$

The eqns. (3) and (7) imply $b=0$, the eqns. (1) and (6) give $a=0$, then according to the eqn. (2) we have $d=0$. Therefore, $D=\left(\begin{array}{ll}0 & 0 \\ c & 0\end{array}\right)$.

$$
\text { Let } A_{6}\left(\alpha_{1}, \beta_{1}\right)=\left(\begin{array}{cccc}
\alpha_{1} & 0 & 0 & 1 \\
\beta_{1} & 1-\alpha_{1} & -\alpha_{1} & 0
\end{array}\right) \text {. }
$$

Then due to eqn. (16) one has the system of equations:

$$
\begin{array}{cl}
a \alpha_{1}-b \beta_{1} & =0, \\
c-3 c \alpha_{1}+2 a \beta_{1}-d \beta_{1} & =0, \\
-b+c+2 b \alpha_{1} & =0, \\
a-a \alpha_{1}+b \beta_{1} & =0, \\
c+2 b \alpha_{1} & =0, \\
-a \alpha_{1}+b \beta_{1} & =0, \\
-a+2 d & =0, \\
b-c-2 b \alpha_{1} & =0 .
\end{array}
$$

The eqns. (1) and (4) imply $a=0$, the eqn. (7) yields $d=0$, the eqns. (3) and (5), imply $b=0$, therefore $c=0$. Hence, $D=0$.

Let $A_{7}\left(\beta_{1}\right)=\left(\begin{array}{cccc}0 & 1 & 1 & 0 \\ \beta_{1} & 1 & 0 & -1\end{array}\right)$. In this case the eqn. (16) is equivalent to the system of equations:

$$
\begin{array}{cl}
2 c-b \beta_{1} & =0, \\
c+2 a \beta_{1}-d \beta_{1} & =0, \\
-b+d & =0, \\
a-2 c+b \beta_{1} & =0, \\
d & =0, \\
-2 c+b \beta_{1} & =0, \\
3 b & =0, \\
b-d & =0 .
\end{array}
$$


We get $b=d=c=a=0$ and therefore, $D=0$.

Let $A=A_{8}\left(\alpha_{1}\right)=\left(\begin{array}{cccc}\alpha_{1} & 0 & 0 & 0 \\ 0 & 1-\alpha_{1} & -\alpha_{1} & 0\end{array}\right)$. Then:

$A_{8}\left(\alpha_{1}\right)(D \otimes I+I \otimes D)-D A_{8}\left(\alpha_{1}\right)=\left(\begin{array}{cccc}a \alpha_{1} & -b+2 b \alpha_{1} & 2 b \alpha_{1} & 0 \\ c-3 c \alpha_{1} & a-a \alpha_{1} & -a \alpha_{1} & b-2 b \alpha_{1}\end{array}\right)$.

We rewrite the eqn. (16) in form of the system of equations as follows:

$$
\begin{array}{cl}
a \alpha_{1} & =0, \\
c-3 c \alpha_{1} & =0, \\
-b+2 b \alpha_{1} & =0, \\
a-a \alpha_{1} & =0, \\
2 b \alpha_{1} & =0, \\
-a \alpha_{1} & =0, \\
b-2 b \alpha_{1} & =0,
\end{array}
$$

The eqns. (1) and (4) imply $a=0$, the eqns. (3) and (5) imply $b=0$ and then $c\left(1-3 \alpha_{1}\right)=0$. If $\alpha_{1} \neq \frac{1}{3}$, one gets $D=\left(\begin{array}{ll}0 & 0 \\ 0 & d\end{array}\right)$, if $\alpha_{1}=\frac{1}{3}$, one has $D=\left(\begin{array}{ll}0 & 0 \\ c & d\end{array}\right)$.

$$
\text { Let } \begin{aligned}
A=A_{9}=\left(\begin{array}{cccc}
\frac{1}{3} & 0 & 0 & 0 \\
1 & \frac{2}{3} & -\frac{1}{3} & 0
\end{array}\right) \text {. Then: } \\
A_{9}(D \otimes I+I \otimes D)-D A_{9}=\left(\begin{array}{cccc}
\frac{a}{3}-b & -\frac{b}{3} & \frac{2 b}{3} & 0 \\
2 a-d & \frac{2 a}{3}+b & -\frac{a}{3}+b & \frac{b}{3}
\end{array}\right) .
\end{aligned}
$$

Due to eqn. (16) one has $a=b=d=0$ and $D=\left(\begin{array}{ll}0 & 0 \\ c & 0\end{array}\right)$.

Let $A=A_{10}=\left(\begin{array}{cccc}0 & 1 & 1 & 0 \\ 0 & 0 & 0 & -1\end{array}\right)$. Then:

$$
A_{10}(D \otimes I+I \otimes D)-D A_{10}=\left(\begin{array}{cccc}
2 c & d & d & 3 b \\
0 & -2 c & -2 c & -d
\end{array}\right) \text {. }
$$

Due to eqn. (16) one has $c=d=b=0$ and $D=\left(\begin{array}{ll}a & 0 \\ 0 & 0\end{array}\right)$.

Let $A=A_{11}=\left(\begin{array}{cccc}0 & 1 & 1 & 0 \\ 1 & 0 & 0 & -1\end{array}\right)$. Then:

$$
A_{11}(D \otimes I+I \otimes D)-D A_{11}=\left(\begin{array}{cccc}
-b+2 c & d & d & 3 b \\
2 a-d & b-2 c & b-2 c & -d
\end{array}\right)=0 .
$$

From the eqn. (16) it is immediate that $D=0$.

Finally, for $A=A_{12}=\left(\begin{array}{llll}0 & 0 & 0 & 0 \\ 1 & 0 & 0 & 0\end{array}\right)$. Then:

$A_{12}(D \otimes I+I \otimes D)-D A_{12}=\left(\begin{array}{cccc}-b & 0 & 0 & 0 \\ 2 a-d & b & b & 0\end{array}\right)=0$, the eqn. (16) gives $D=\left(\begin{array}{cc}a & 0 \\ c & 2 a\end{array}\right)$.
Now we present the corresponding results for characteristic 2 and 3 cases without any justifications as far as they are similar to that of the proofs in $\operatorname{Char}(\mathbb{F}) \neq 2,3$ case above.

\section{Theorem 4.2}

The derivations of all the algebras on 2-dimensional vector space over an algebraically closed field $\mathbb{F}$ of characteristic 2 are given as follows.

$$
\begin{aligned}
& \operatorname{Der}\left(A_{1,2}\left(\alpha_{1}, \alpha_{2}, \alpha_{4}, \beta_{1}\right)\right)=\{0\}, \\
& \operatorname{Der}\left(A_{2,2}\left(\alpha_{1}, \beta_{1}, \beta_{2}\right)\right)=\{0\} \text { if } \beta_{1} \neq 0, \operatorname{Der}\left(A_{2,2}\left(\alpha_{1}, 0, \beta_{2}\right)\right)=\left\{\left(\begin{array}{ll}
0 & 0 \\
0 & d
\end{array}\right): d \in \mathbb{F}\right\} \text {, } \\
& \operatorname{Der}\left(A_{3,2}\left(\beta_{1}, \beta_{2}\right)\right)=\{0\} \text { if } \beta_{2} \neq 1, \operatorname{Der}\left(A_{3,2}\left(\beta_{1}, 1\right)\right)=\left\{\left(\begin{array}{ll}
0 & 0 \\
c & 0
\end{array}\right): c \in \mathbb{F}\right\}, \\
& \operatorname{Der}\left(A_{4,2}\left(\alpha_{1}, \beta_{2}\right)\right)=\left\{\left(\begin{array}{ll}
0 & 0 \\
0 & d
\end{array}\right): d \in \mathbb{F}\right\} \text { if } \beta_{2} \neq 1, \\
& \operatorname{Der}\left(A_{4,2}\left(\alpha_{1},-1\right)\right)=\left\{\left(\begin{array}{ll}
0 & 0 \\
c & d
\end{array}\right): c, d \in \mathbb{F}\right\}, \\
& \operatorname{Der}\left(A_{5,2}\left(\alpha_{1}\right)\right)=\left\{\left(\begin{array}{ll}
0 & 0 \\
c & 0
\end{array}\right): c \in \mathbb{F}\right\} \text {, } \\
& \operatorname{Der}\left(A_{6,2}\left(\alpha_{1}, \beta_{1}\right)\right)=\{0\} \text { if } \beta_{1} \neq 0, \operatorname{Der}\left(A_{6,2}\left(\alpha_{1}, 0\right)\right)=\left\{\left(\begin{array}{ll}
0 & 0 \\
0 & d
\end{array}\right): d \in \mathbb{F}\right\} \text {, } \\
& \operatorname{Der}\left(A_{7,2}\left(\alpha_{1}\right)\right)=\{0\} \text { if } \alpha_{1} \neq 1, \operatorname{Der}\left(A_{7,2}(1)\right)=\left\{\left(\begin{array}{ll}
0 & 0 \\
c & 0
\end{array}\right): c \in \mathbb{F}\right\} \text {, } \\
& \operatorname{Der}\left(A_{8,2}\left(\alpha_{1}\right)\right)=\left\{\left(\begin{array}{ll}
0 & 0 \\
0 & d
\end{array}\right): d \in \mathbb{F}\right\} \text { if } \alpha_{1} \neq 1, \operatorname{Der}\left(A_{8,2}(1)\right)=\left\{\left(\begin{array}{ll}
0 & 0 \\
c & d
\end{array}\right): c, d \in \mathbb{F}\right\} \text {, } \\
& \operatorname{Der}\left(A_{9,2}\right)=\left\{\left(\begin{array}{ll}
0 & 0 \\
c & 0
\end{array}\right): c \in \mathbb{F}\right\}, \operatorname{Der}\left(A_{10,2}\right)=\left\{\left(\begin{array}{ll}
a & 0 \\
c & 0
\end{array}\right): a, c \in \mathbb{F}\right\} \text {, } \\
& \operatorname{Der}\left(A_{11,2}\right)=\{0\}, \operatorname{Der}\left(A_{12,2}\right)=\left\{\left(\begin{array}{ll}
a & 0 \\
c & 0
\end{array}\right): a, c \in \mathbb{F}\right\} \text {. }
\end{aligned}
$$

\section{Theorem 4.3}

The derivation of all the algebras on 2-dimensional vector space over an algebraically closed field $\mathbb{F}$ of characteristic 3 are given as follows.

$$
\begin{aligned}
& \operatorname{Der}\left(A_{1,3}\left(\alpha_{1}, \alpha_{2}, \alpha_{4}, \beta_{1}\right)\right)=\operatorname{Der}\left(A_{2,3}\left(\alpha_{1}, \beta_{1}, \beta_{2}\right)\right)=\operatorname{Der}\left(A_{3,3}\left(\beta_{1}, \beta_{2}\right)\right)=\{0\}, \\
& \operatorname{Der}\left(A_{4,3}\left(\alpha_{1}, \beta_{2}\right)\right)=\left\{\left(\begin{array}{ll}
0 & 0 \\
0 & d
\end{array}\right): d \in \mathbb{F}\right\} \text { if } \beta_{2} \neq 2 \alpha_{1}-1, \\
& \operatorname{Der}\left(A_{4,3}\left(\alpha_{1}, 2 \alpha_{1}-1\right)\right)=\left\{\left(\begin{array}{ll}
0 & 0 \\
c & d
\end{array}\right): c, d \in \mathbb{F}\right\}, \operatorname{Der}\left(A_{5,3}\left(\alpha_{1}\right)\right)=\left\{\left(\begin{array}{ll}
0 & 0 \\
c & 0
\end{array}\right): c \in \mathbb{F}\right\}, \\
& \operatorname{Der}\left(A_{6,3}\left(\alpha_{1}, \beta_{1}\right)\right)=\operatorname{Der}\left(A_{7,3}\left(\beta_{1}\right)\right)=\{0\}, \operatorname{Der}\left(A_{8,3}\left(\alpha_{1}\right)\right)=\left\{\left(\begin{array}{ll}
0 & 0 \\
0 & d
\end{array}\right): d \in \mathbb{F}\right\}, \\
& \operatorname{Der}\left(A_{9,3}\right)=\left\{\left(\begin{array}{ll}
0 & 2 c \\
c & 0
\end{array}\right): c \in \mathbb{F}\right\}, \operatorname{Der}\left(A_{10,3}\right)=\left\{\left(\begin{array}{ll}
a & b \\
0 & 0
\end{array}\right): a, b \in \mathbb{F}\right\}, \\
& \operatorname{Der}\left(A_{11,3}\right)=\left\{\left(\begin{array}{ll}
0 & 0 \\
c & 0
\end{array}\right): c \in \mathbb{F}\right\}, \operatorname{Der}\left(A_{12,3}\right)=\left\{\left(\begin{array}{ll}
a & 0 \\
c & 2 a
\end{array}\right): a, c \in \mathbb{F}\right\} .
\end{aligned}
$$

\section{Remark 4.4}

The sets of the algebras of types $A_{1}\left(\alpha_{1}, \alpha_{2}, \alpha_{4}, \beta_{1}\right), A_{1,2}\left(\alpha_{1}, \alpha_{2}, \alpha_{4}, \beta_{1}\right), A_{1,3}\left(\alpha_{1}, \alpha_{2}, \alpha_{4}, \beta_{1}\right)$ are open, dense subsets of $V=M\left(\mathrm{~mm}^{2} ; \mathbb{F}\right)$ and therefore due to the results presented we can conclude that the majority of 2-dimensional algebras have only trivial automorphism groups and derivation algebras.

\section{Conclusion}

The automorphism groups and derivation algebras of all two- 
Citation: Ahmed H, Bekbaev U, Rakhimov I (2018) The Automorphism Groups and Derivation Algebras of Two-Dimensional Algebras. J Generalized Lie Theory Appl 12: 290. doi: 10.4172/1736-4337.1000290

Page 9 of 9

dimensional algebras over algebraically closed fields are described through the all theorems and proofs.

\section{Acknowledgment}

The first and third authors acknowledge UPM for a support by GP-IPS 9537100 and the second author's research was supported by FRGS14-153-0394, MOHE.

\section{References}

1. Shestakov I, Umirbaev U (2004) The tame and the wild automorphisms of polynomial rings in three variables. J Amer Math Soc 17: 197-227.

2. Alev J, Chamarie M (1992) Dérivations et automorphismes de quelques al'gebres quantiques. Comm Algebra 20: 1787-1802.

3. Alev J, Dumas F (1996) Rigidité des plongements des quotients primitifs minimaux de $U q(s /(2))$ dans algébre quantique de Weyl-Hayashi Nagoya. Math J 143: 119-146.

4. Andruskiewitsch $\mathrm{N}$, Dumas $\mathrm{F}(2008)$ On the automorphisms of $U_{q}^{+}(g)$, in Quantum groups. IRMA Lect Math Theor Phys 12:107-133.

5. Bavula VV, Jordan DA (2001) Isomorphism problems and groups of automorphisms for generalized Weyl algebras. Trans Amer Math Soc 353 769-794.
6. Gómez-Torrecillas J, El Kaoutit L (2002) The group of automorphisms of the coordinate ring of quantum symplectic space. Beiträge Algebra Geom 43: 597601.

7. Suárez-Alvarez M, Vivas Q (2012) Automorphisms and isomorphism of quantum generalized Weyl algebras, preprint, arXiv:1206.4417v1.

8. Yakimov M (2013) The Launois-Lenagan conjecture. J Algebra 392: 1-9.

9. Yakimov M (2014) Rigidity of quantum tori and the Andruskiewitsch-Dumas conjecture. Selecta Math 20: 421-464.

10. Goodearl KR, Yakimov MT (2013) Unipotent and Nakayama automorphisms of quantum nilpotent algebras, preprint, arXiv:1311.0278.

11. Ceken S, Palmieri JH, Wang YH, Zhang JJ (2015) The discriminant controls automorphism groups of noncommutative algebras. Advances in Mathematics 269: 551-584.

12. Ahmed H, Bekbaev U, Rakhimov I (2017) Complete classification of two dimensional algebras, AIP Conference Proceedings 1830: 070016.

13. Kaygorodov I, Volkov Y (2017) The variety of 2-dimensional algebras over an algebraically closed field, ArXiv: 1701.08233math.RA pp: 1-15. 